\title{
Jörg Kleis: African Regional Community Courts and their Contribution to Continental Integration.
}

\author{
Baden-Baden (Nomos), 2016, 391 S., $99 €$ (Recht und Verfassung in Afrika, \\ Band 29 - Law and Constitution in Africa, vol. 29)
}

\author{
Besprochen von Privatdozent Dr. Harald Sippel, Bayreuth
}

In seiner 2016 vom Fachbereich Rechtswissenschaft der Freien Universität Berlin angenommenen juristischen Dissertation befasst sich Jörg Kleis mit der Frage, welchen Beitrag die von afrikanischen Staaten errichteten gemeinschaftlichen Gerichtshöfe zur regionalen Integration der teilnehmenden Staaten zu leisten vermögen.

Nach dem einleitenden Kapitel, in dem der Verfasser seine Untersuchungsmethode vorstellt und mit Hinweis auf den Gerichtshof der Europäischen Union in Luxemburg darlegt, dass die Rechtsprechung eines gemeinschaftlichen internationalen Spruchkörpers sehr wohl zur regionalen Integration beiträgt, verfolgt er im zweiten Abschnitt die Entwicklung der regionalen Integration in Afrika in ökonomischer, politischer und rechtlicher Hinsicht am für seine Doktorschrift zentralen Beispiel der Westafrikanischen Wirtschaftsgemeinschaft (Economic Community of West African States - ECOWAS), der Entwicklungsgemeinschaft des südlichen Afrika (Southern African Development Community - SADC) sowie der Ostafrikanischen Gemeinschaft (East African Community - EAC). Diese internationalen Organisationen errichteten mit dem Gerichtshof der ECOWAS (Community Court of Justice) im nigerianischen Abuja, dem SADC-Gerichtshof (SADC Tribunal) im namibischen Windhoek und dem Ostafrikanischen Gerichtshof (East African Court of Justice) im tansanischen Arusha Gemeinschaftsgerichte, deren Personal, Verwaltung und finanzielle Ausstattung Gegenstand des dritten Kapitels sind.

In den nachfolgenden fünf Abschnitten behandelt der Verfasser ausführlich wesentliche Probleme, denen Gemeinschaftsgerichte im Rahmen ihrer rechtsprechenden Tätigkeit ausgesetzt sind. So vermitteln etwa die Frage der anzuwendenden Gerichtssprache und der Hinweis auf unterschiedliche Methoden der Entscheidungsfindung Einblicke in verschiedenartige afrikanische Rechtskulturen. Breiten Raum nimmt die Erörterung der in internationalen Verträgen festgelegten Zuständigkeiten der Gemeinschaftsgerichte und die dazu erlassenen wegweisenden Gerichtsentscheidungen ein, welche der Verfasser ebenso vorstellt und analysiert wie einzelne gemeinschaftsgerichtliche Urteile zur Anwendung und Auslegung von Gemeinschaftsrecht. Auffällig sei dabei, dass bei allen untersuchten Wirtschaftsgemeinschaften nicht selten nationale Befindlichkeiten einzelner afrikanischer Staaten eine eigentlich auch in rechtlicher Hinsicht angestrebte regionale Integration beeinträchtigen. Dies führe bisweilen sogar zur Missachtung von gemeinschaftsgerichtlichen Entscheidungen durch Mitgliedstaaten, ein Umstand, den der Verfasser als besonders integrationswidriges Verhalten erachtet und ihn veranlasst, in Anlehnung an Regelungen der Europäischen 
Union zur Durchsetzung von gemeinschaftsrechtlichen Maßnahmen und Entscheidungen Vorschläge zu unterbreiten, welche geeignet sind, gemeinschaftsgerichtlichen Urteilen auch in den afrikanischen Wirtschaftsgemeinschaften zur Geltung zu verhelfen. Da sich diese internationalen Organisationen vertraglich sämtlich zur Einhaltung der Menschenrechte verpflichtet haben, richtet der Verfasser sein besonderes Augenmerk auf die Frage, inwieweit die regionale Integration in Afrika durch den gemeinschaftsgerichtlichen Schutz der Menschenrechte gefördert werden kann, und erkennt in der bislang vorliegenden Rechtsprechung sogar erste Anzeichen für einen gesamtafrikanischen Menschenrechtsschutz. Resümierend stellt er dazu fest, dass die in Afrika gewünschte wirtschaftliche Integration nur erreicht werden kann, wenn die Einhaltung der Menschenrechte in den einzelnen Mitgliedstaaten der Wirtschaftsgemeinschaften gewährleistet ist.

Die im neunten und letzten Kapitel enthaltenen Schlussfolgerungen bieten eine ausführliche Zusammenfassung der Untersuchungsergebnisse, unterbreiten Vorschläge zur Intensivierung der regionalen Integration mittels Stärkung der Gemeinschaftsgerichte und geben in dieser Hinsicht einen vorsichtigen Ausblick auf mögliche künftige Entwicklungen in Afrika, die idealerweise einer „kontinentalen Integration“ Vorschub leisten.

Das bereits im Titel der Doktorschrift zum Ausdruck gekommene Ziel der „kontinentalen Integration“ ist indes sehr ambitioniert. Zwar räumt der Verfasser ein, dass sich seine Untersuchung auf die südlich der Sahara gelegenen afrikanischen Staaten bezieht und somit die nordafrikanischen Länder ausspart, jedoch verbleibt neben den 36 Staaten, die an den drei vorgenannten afrikanischen Wirtschaftsgemeinschaften beteiligt sind (ECOWAS: 15; SADC: 16; EAC: 6, unter Berücksichtigung des Kuriosums der simultanen Mitgliedschaft Tansanias sowohl in der SADC als auch in der EAC) rund ein Dutzend weitere afrikanische Länder, die bislang überhaupt keiner Wirtschaftsgemeinschaft angehören und daher auch nicht an einem Integrationsprozess teilhaben können. Dabei gilt es zu berücksichtigen, dass die Rechtsprechung des Afrikanischen Menschenrechtsgerichtshofs (African Court on Human and Peoples ' Rights) im tansanischen Arusha nicht Gegenstand der Untersuchung ist.

Im Weiteren ist es durchaus fraglich, ob die Entscheidungen der afrikanischen Gemeinschaftsgerichte eine Integrationswirkung für den gesamten Kontinent entfalten können, insbesondere unter Berücksichtigung des Umstandes, dass der Gerichtshof der ECOWAS von 2001 bis zum Abschluss der Dissertation lediglich 122 Entscheidungen, der Ostafrikanische Gerichtshof seit 2002 nur 78 Entscheidungen und der SADC-Gerichtshof seit 2005 gerade einmal 20 Entscheidungen (S. 98-109) ausgefertigt haben, viele davon mit engem Bezug zu den für die jeweilige Wirtschaftsgemeinschaft relevanten Verträgen. Dies erschwert nicht nur eine Vergleichbarkeit der Entscheidungen, sondern erst recht eine mögliche Übertragbarkeit der Rechtsprechung und eine Kooperation der Gerichtshöfe. Überhaupt findet, wie der Verfasser ausführt, eine Entwicklung des Gemeinschaftsrechts in afrikanischen Staaten weniger über die Gerichte als über den gemeinschaftlichen Gesetzgeber statt, der insbesondere auf die Harmonisierung der bestehenden nationalen Gesetze hinwirkt (S. 360).

Wie fragil der Bestand eines afrikanischen Gemeinschaftsgerichts sein kann, wenn der politische Wille einzelner Mitgliedstaaten zu einer gemeinsamen Rechtsprechung fehlt, 
zeigt das vom Verfasser dargelegte Beispiel des SADC-Gerichtshofs. Dieser wurde zwar bereits 1992 gegründet, jedoch erst 2005 mit der Ernennung der Mitglieder des Gerichts eingerichtet, um seine Arbeit schon nach weiteren fünf Jahren einzustellen, da einige seiner Urteile, die den Schutz der Menschenrechte zum Gegenstand hatten, von dem Mitgliedstaat Simbabwe missachtet wurden. Nach einer 2012 erfolgten Reform mit einer Beschränkung seiner Aufgaben auf zwischenstaatliche Fälle ist der SADC-Gerichtshof seither für die Rechtsentwicklung in Afrika nahezu bedeutungslos geworden. Es sei daher durchaus ernüchternd, wenn sich nicht einmal die Staaten im südlichen Afrika auf den gemeinschaftsgerichtlichen Menschenrechtsschutz einigen könnten und es sogar vorzögen, den Gerichtshof ihrer Gemeinschaft zu entmachten (S. 353). Für eine vom Verfasser angedachte „kontinentale Integration“ ist der Weg daher noch weit.

Hilfreich sind die Verzeichnisse der rechtlichen Materialien zu den drei untersuchten afrikanischen Wirtschaftsgemeinschaften und der verwendeten Entscheidungen der Gemeinschaftsgerichtshöfe sowie etlicher nationaler Gerichte in Afrika, ebenso wie die ausführlichen bibliographischen Hinweise. Die Dissertation von Jörg Kleis ist ein klar strukturiertes, gründlich recherchiertes, informatives und sehr gut lesbares Werk, das sich an alle an den regionalen Integrationsprozessen in Afrika und ihren judiziellen Implikationen interessierten Personen richtet. 